\title{
Interpretation of Energy-Filtered BSE Images at Ultra Low Voltage Conditions
}

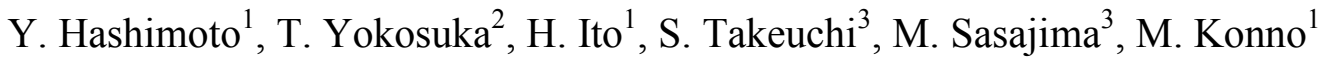

1. Application Development Department, Science \& Medical Systems Design Division, Hitachi

High-Technologies, 1040, Ichige, Hitachinaka, Ibaraki, 312-0033, Japan

2. 1st Department, Research and Development Division, Hitachi High-Technologies, 882, Ichige, Hitachinaka, Ibaraki, 312-8504, Japan

3. Advanced Microscope Systems Design Department, Science \& Medical Systems Design Division, Hitachi High-Technologies, 882, Ichige, Hitachinaka, Ibaraki, 312-8504, Japan

To meet the increasing demands to clarify the compositional differences in advanced composite materials, we report a new imaging method using energy filtered BSE signal at Ultra Low Voltage (ULV)[1]. This method was applied to a carbon nanotube (CNT) and polytetrafluoroethylene (PTFE) composite film to confirm its ability to differentiate distribution of carbon nanotube (CNT) and polytetrafluoroethylene (PTFE) components at $300 \mathrm{~V}$ as shown in Fig The results encouraged us to further explore the method's extensive imaging capability for differentiating materials with similar atomic number that were previously not differentiated by the conventional theory of SEM signal behavior. However, our investigation of the observed effects continues, in order to better understand the different BSE signal intensity between CNT and PTFE under the new method's conditions. In this study, we investigated the BSE signal behavior at Ultra low voltage from fundamental experiments using the SU8200 series FE-SEM and electron simulation techniques.

In the CNT/PTFE sample, BSE signal intensity is considered to be affected by 3 factors; difference of average atomic number, surface morphology and surface potential. Here we focused on the relationship between surface potential and BSE signal intensity. Figure 2 shows the simplified model for the simulation experiment. The model consists of a detector plane, vacuum space and carbon specimen surface. A voltage from 0 to $-100 \mathrm{~V}$ was set at the specimen surface with $20 \mathrm{~V}$ step. BSE trajectory was detected at the detector plane which was located $100 \mu \mathrm{m}$ above the specimen surface.

Figure 3 shows BSE signal intensity against detection angle in different surface potentials given by simulation results at $0.3 \mathrm{kV}$. According to the result of electron trajectory simulation, the angle range of BSE detected by the top detector was from 60 degrees to 80 degrees as shown by the blue area in Figure 3 . If the surface potential was $0 \mathrm{~V}$, the BSE angle peak was about 45 degrees. When the surface negative potential was increased, the peak of the signal intensity moved to higher angles since the BSE trajectory is affected by the potential as shown in Fig. 4.

Based on the above described experimental result, we theorized that the contrast between CNT and PTFE is caused by surface potential. PTFE is a well-known insulating material, while CNT is a conductor. According to the simulation's results, if the PTFE is negatively charged then it produces brighter contrast than the CNT. We will disclose more details in the session.

Acknowledgement

The CNT / PTFE composite film specimen was kindly supplied by Prof. Yoshiyuki SHOW of the Department of Electrical and Electronic Engineering, School of Engineering, Tokai University. 


\section{Reference}

[1] Y. Hashimoto et al., Microsc. Microanal. 19 (Suppl 2), 1176-1177 (2013).
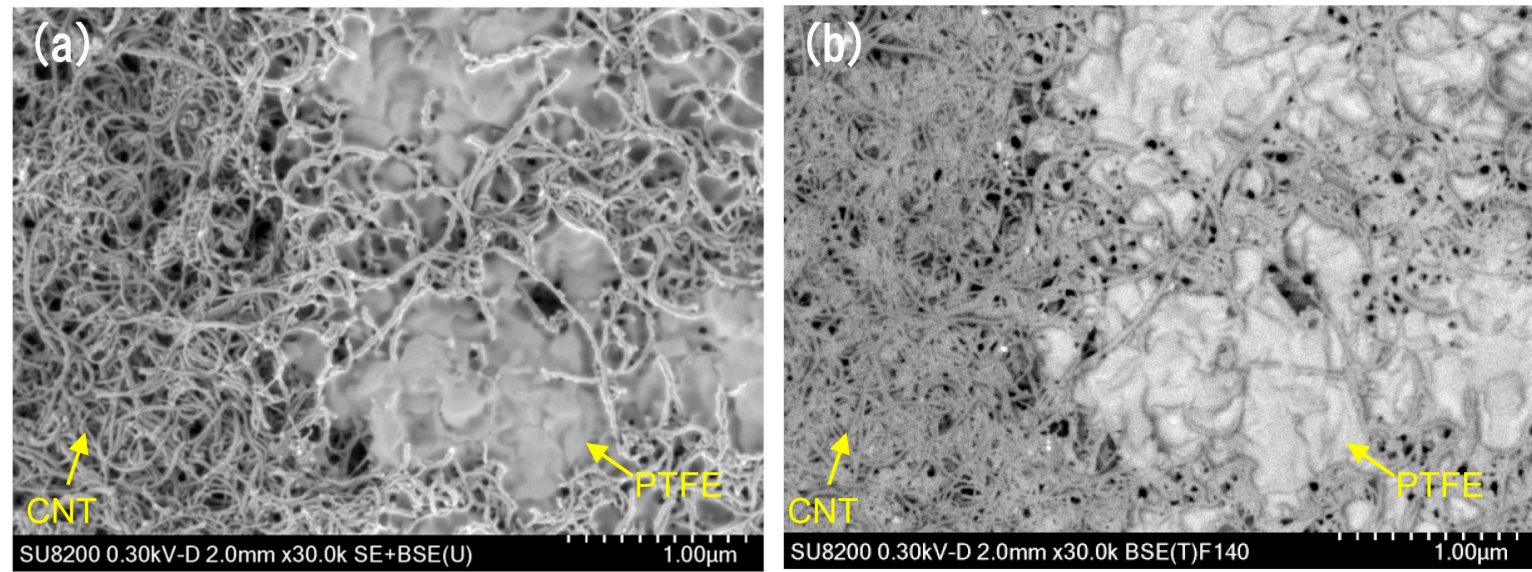

Fig.1 BSE images of CNT / PTFE composite film detected by (a)Upper detector, (b)Top detector

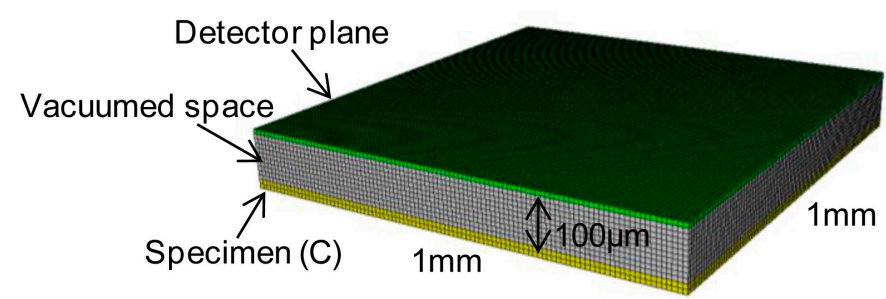

Fig.2 Simplified model of charging simulation

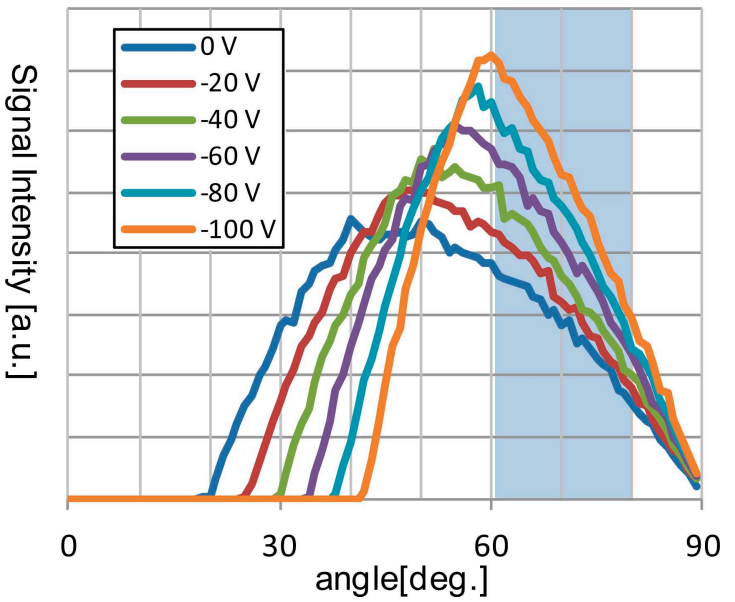

Fig.3 Distribution of BSE angle

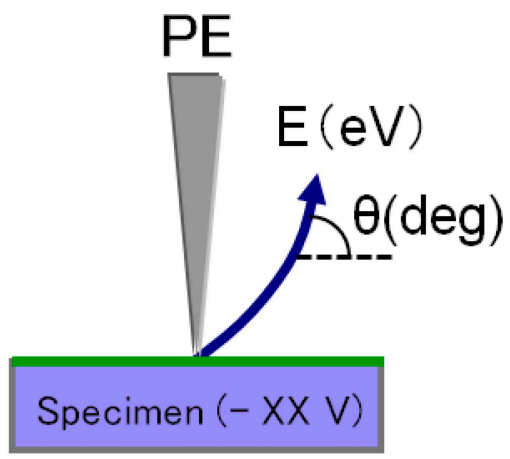

(b) (a)

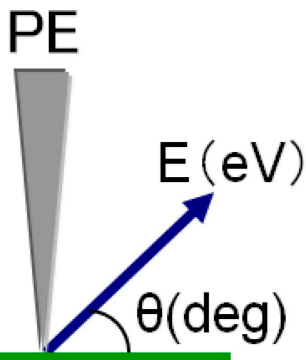

Specimen (0 V)

Fig. 4 Schematics of effect of surface potential to electron trajectory (Surface potential is (a) $0 \mathrm{~V},(\mathrm{~b})-\mathrm{XX} \mathrm{V}$ ) 